\title{
Caracterización de las cibercomunidades de aprendizaje (cCA)
}

\section{Characterization of Learning Cybercommunities}

\author{
Iñaki Murua Anzola \\ Helduen Alfabetatzerako eta Berreuskalduntzerako Erakundea - Instituto para la Alfabetización y \\ Reeuskaldunización de Adultos (HABE). España. \\ i-murua@habe.eus \\ Domingo J. Gallego Gil \\ Universidad Nacional de Educación a Distancia (UNED). España. \\ dgallego@edu.uned.es \\ María Luz Cacheiro González \\ Universidad Nacional de Educación a Distancia (UNED). España. \\ mlcacheiro@edu.uned.es
}

\begin{abstract}
Resumen
En este artículo se presentan los principales resultados de la investigación exploratoria llevada a cabo sobre las cibercomunidades de aprendizaje (cCA) y la formación del profesorado aplicando, entre otros, el método Delphi; el marco teórico y contextual de la misma se presentó en el número 43 de la revista RED. Los participantes del panel de expertos han sido 31 encuestados online con el instrumento Delphi-cCA, y 19 responsables de comunidades que han contestado al cuestionario-cCA. Entre los principales resultados se destacan las condiciones para la creación y desarrollo de cCA, las herramientas y funcionalidades de las comunidades y las tipologías de participación (personas implicadas, activas, pasivas y ausentes).
\end{abstract}

\section{Palabras clave}

Cibercomunidades de aprendizaje, comunidades de práctica, comunidades virtuales, Método Delphi, formación del profesorado.

\begin{abstract}
This article presents the main results of the exploratory research conducted on learning cybercommunities (cCA) and teacher training using, among others, the Delphi method. The theoretical and contextual framework was presented at RED Journal number 43. The expert panel included 31 experts surveyed online with Delphi-CCA instrument, and 19 responsibles for learning communities who responded to the questionnaire-cCA. Among the main results, the conditions for the creation and development of cCA, tools and functionalities of the communities, and the types of participation (involved, active, passive, and absent) stand out.
\end{abstract}

\section{Key words}

Learning cybercommunities, communities of practice, virtual communities, Delphi Method, teacher training.

\section{Introducción y marco teórico}

Las personas han pertenecido siempre a comunidades en las que han ido aprendiendo "de" y "con" otros miembros. Ahora bien; en la medida que Internet ha supuesto una ruptura de las coordenadas espacio-temporales en las que nos movemos, parece que deben valorarse las posibilidades de los agregados sociales que surgen en la Red (Rheingold, 1996) en ámbitos como la formación, en general, y la formación del profesorado, en particular. 
Así, existen trabajos que ayudan a comprender la estructura de una comunidad de aprendizaje en el ciberespacio (Tirado y Martínez, 2009 y Rovai, 2002), pero, en todo caso, no conviene obviar que existen peligros (García Aretio, 2003), limitaciones en el uso de las TIC para el trabajo grupal y cooperativo (Gros, García y Lara, 2009), dudas con aspectos tales como la participación asimétrica (Murua y Gallego, 2014; Park, 2015), el liderazgo, el languidecimiento y decaimiento tras la ilusión inicial o que las comunidades no sean en sí mismo positivas (Escudero, 2009).

En relación a la base teórica de la investigación sobre formación del profesorado a través de cibercomunidades de aprendizaje (cCA) (Murua, Cacheiro y Gallego, 2014), nos encontramos en un primer lugar con una necesidad de clarificación conceptual. En efecto; tal y como señalan distintos autores, como Gros et al. (2009) o Coll, Bustos y Engel (2008, hay términos, "comunidades de aprendizaje" o "comunidades virtuales" entre otros, que son de referencia frecuente en la bibliografía y que han alcanzado un nivel de difusión en la sociedad. Sin embargo, es constatable que se utilizan distintas denominaciones para nombrar realidades semejantes (sinonimias) o que aunque se enfatice en la idea del aprendizaje como construcción social, haya diferentes planteamientos subyacentes aún cuando se utilice un nombre similar (polisemias).

Si bien "comunidad virtual" sea el más extendido en la bibliografía, se ha optado por utilizar "cibercomunidad", siguiendo a García Aretio (2003). Pese a que el adjetivo "virtual" también se utiliza para referirse a organizaciones, comunidades, actividades y prácticas que tienen lugar en Internet, si se contrapone "virtual" a "real", entendemos las cibercomunidades como reales, constituidas por personas físicas, y por tanto reales, y no como deficitarias respecto a las presenciales. Así, llegamos a una primera definición de cCA tras la revisión bibliográfica realizada: son grupos de personas (profesionales, estudiantes, gentes con intereses comunes...) comunicadas / vinculadas a través de redes / Internet de forma continuada, para compartir información, ideas, reflexiones, experiencias... con el interés común de velar por su desarrollo personal, académico y profesional, y el de los demás miembros que la componen. Tal y como se desprende de la presente definición, estamos más cerca del concepto de "comunidad de práctica" (Draper, 2015) y no del movimiento de las comunidades de aprendizaje (Flecha y Larena, 2008).

En relación a las orientaciones y criterios para el estudio, diseño y desarrollo de las cCA, nos hemos fijado en tres aspectos principales. En primer lugar, en la revisión y caracterización de los principios y estrategias que pueden constituir o constituyen de facto la base para el estudio, creación y desarrollo citados. En segundo, en las herramientas y funcionalidades utilizadas en las cCA de entre las posibilidades que se van dando en el tiempo en el campo de las TIC. Por último, en el modo de participación de los miembros de la cCA.

\section{Objetivos}

Los objetivos planteados en la investigación han sido:

- Delimitar conceptualmente una cCA con un enfoque integrador.

Caracterización de las cibercomunidades de aprendizaje. Iñaki Murua Anzola, Domingo J. Gallego Gil y María Luz Cacheiro González. 
- Identificar las condiciones de creación y desarrollo de una cCA con personas adultas, más allá del ámbito de la educación formal o de procesos formativos estructurados.

- $\quad$ Establecer la importancia concedida a las herramientas / funcionalidades en una cCA y su nivel de uso.

- Valorar el nivel de participación en las cCA.

- Elaborar una propuesta de cCA para la formación del profesorado en contextos no formales e informales.

\section{Metodología y contexto de la investigación}

En cuanto al marco de referencia metodológico, podemos decir que se ha tratado de un estudio exploratorio, con un enfoque mixto cualitativo-cuantitativo (Cohen y Manion, 1990; Hernández Sampieri, Fernández Collado y Baptista, 2010).

Se han utilizado distintos procedimientos metodológicos. Junto a análisis documental, observaciones y diario electrónico (Domínguez Figaredo, 2003), cabe destacar la aplicación del método Delphi (Cabero, 2014; Cabero y Barroso, 2013; Landeta, 1999; Luna, Infante, Martínez López y García Ordaz, 2004), así como un cuestionario a responsables de cibercomunidades puestas como ejemplo por el panel de expertos del Delphi o detectadas durante las búsquedas bibliográficas y en Internet. El análisis documental, a su vez, ha estado retroalimentado por los datos que se iban obteniendo de las sucesivas rondas del Delphi-cCA.

Tanto el cuestionario aplicado al panel de expertos (Delphi-cCA), como el enviado a los responsables de comunidades (Cuestionario-cCA), se validaron mediante prueba de jueces, con carácter previo a su utilización. Indicar, asimismo, que ambos cuestionarios tenían preguntas cerradas y abiertas.

Con referencia al proceso del Delphi (Ilustración 1) se han llevado a cabo tres rondas -criterio que estaba inicialmente previsto para la finalización del proceso iterativodesde junio a octubre de 2014. Pueden distinguirse en el mismo las tres fases (preliminar, exploratoria y final) que proponen Blasco, López Padrón y Mengual (2010). 


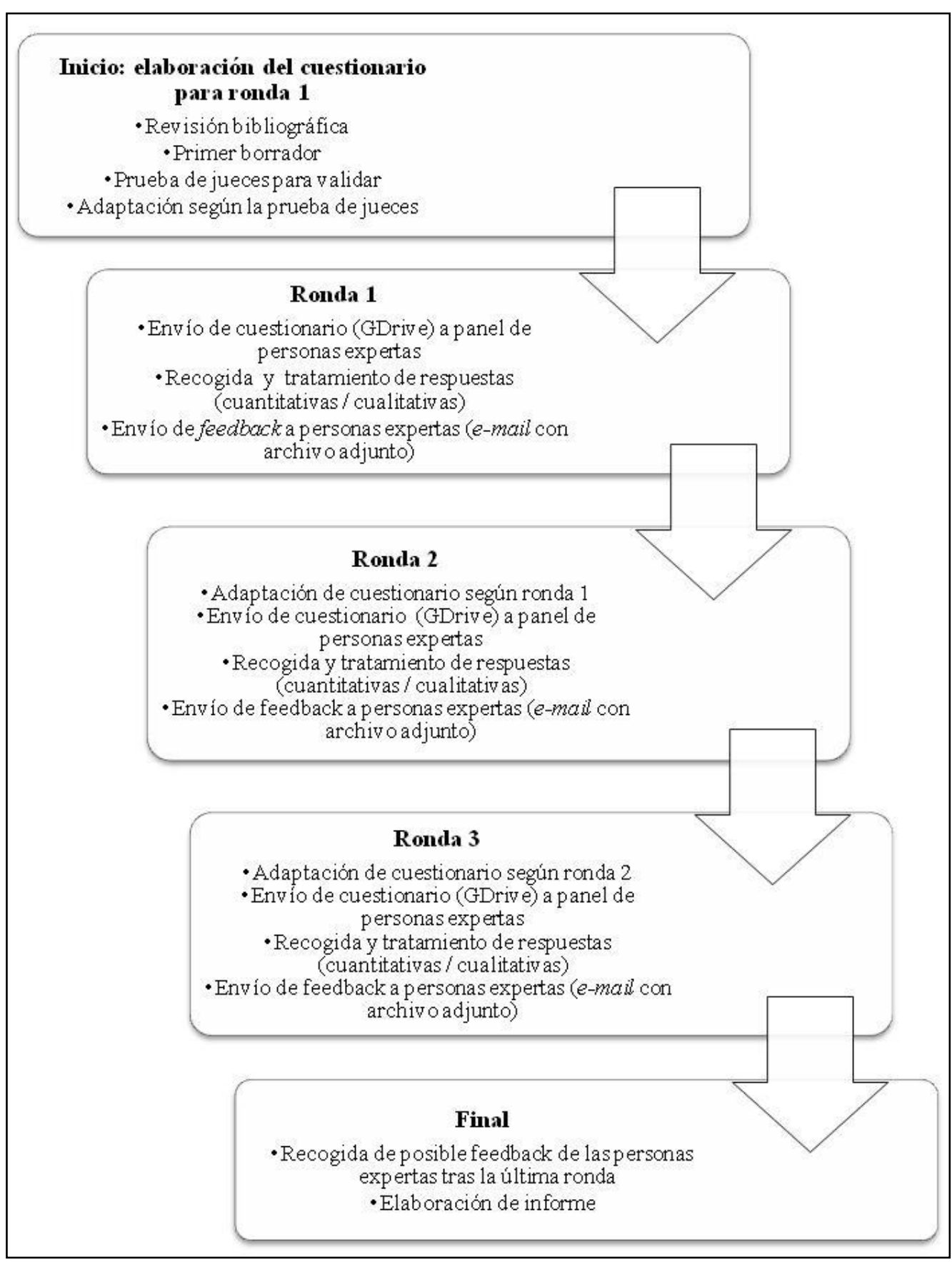

\section{Ilustración 1. Proceso para el Delphi-cCA}

Se ha contado con la participación de 31 personas expertas en total, seleccionadas a través de su biograma y con un nivel establecido de expertía, calculada según el coeficiente de competencia experta K (Cabero y Barroso, 2013; Cabero y Llorente, 2013); en la Ilustración 2 se representa el proceso seguido para la constitución del panel de personas expertas, considerada una de las claves para el éxito de un Delphi.

En relación a los criterios establecidos con carácter previo para elaborar la lista de posibles participantes se consideró que los expertos no lo fueran en el ámbito exclusivamente académico o de la educación en sus distintos niveles, sino ampliar el 
punto de vista con otro tipo de agentes que también llevaran a cabo su tarea en el campo de las comunidades, tal y como pueden ser las redes sociales profesionales. De ahí que se haya optado por la participación de distintos expertos del ámbito hispano, que respondieran a este perfil general:

- $\quad$ Creadores o responsables de distintos grupos formados en Internet.

- Investigadores en y sobre entornos virtuales, comunidades, redes sociales...

- $\quad$ Personas de reconocido prestigio en el ámbito de Internet.

Atendiendo a dichos criterios, se ha procedido la elaboración de una lista previa de 50 personas expertas.

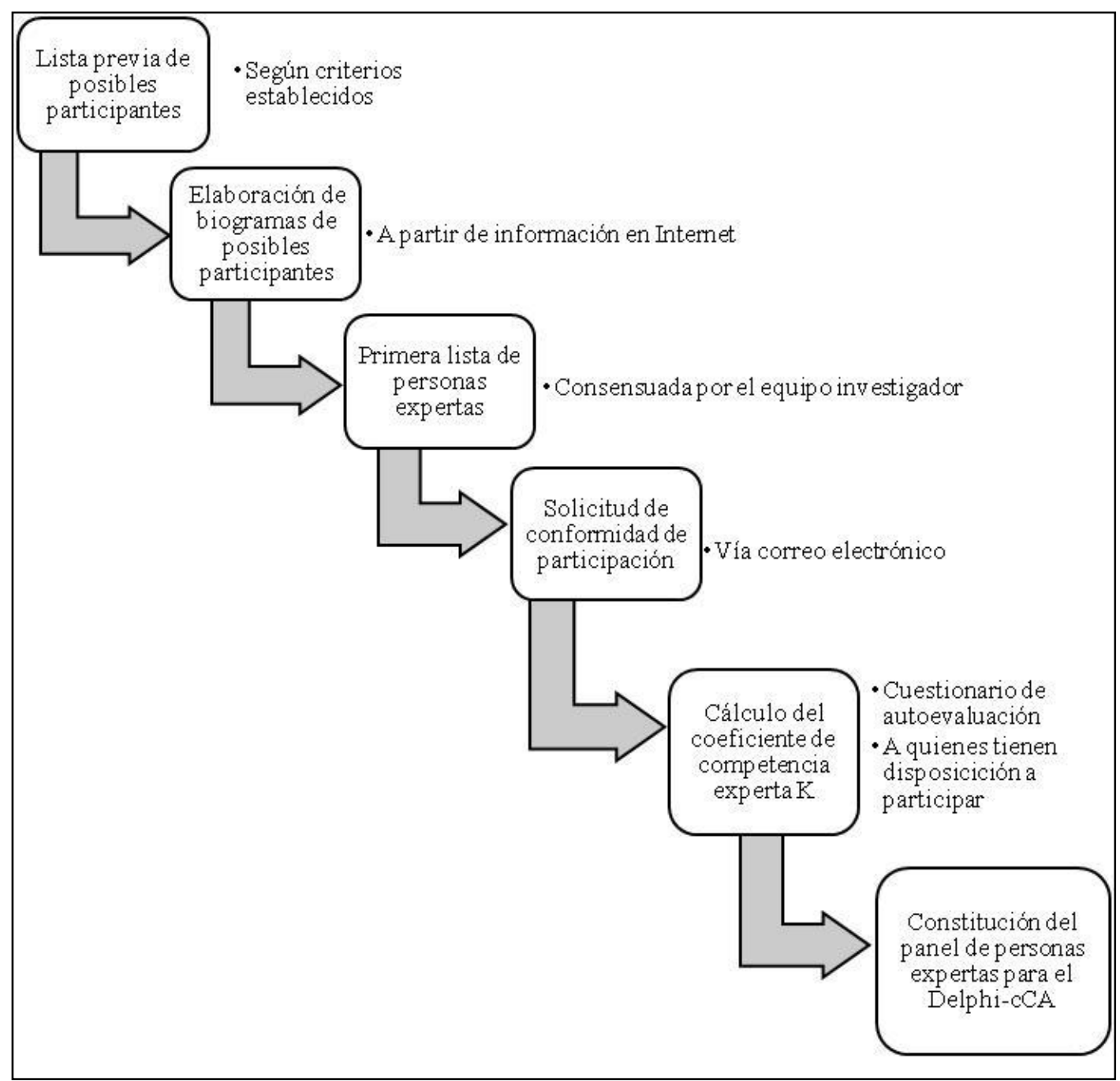

Ilustración 2. Proceso para la constitución del panel de personas expertas para el Delphi-cCA

En relación al cuestionario-cCA, el muestreo también ha sido intencional, aplicando el proceso de "bola de nieve" (Hernández-Sampieri et al., 2010). Se ha invitado a participar a responsables / miembros destacados de cibercomunidades que se han dado como ejemplo de buenas prácticas en el proceso seguido en las tres rondas del Delphi- 
cCA; asimismo se ha incluído a otras comunidades encontradas en las búsquedas bibliográficas y de Internet (Murua, 2015), obteniendo una respuesta favorable de 19 responsables de comunidades de aprendizaje.

\section{Resultados}

De las 31 personas que han compuesto el panel de expertos, 25 han participado en las tres rondas, siendo 16 quienes han respondido a todos y cada uno de los ítems propuestos. Aunque el criterio preestablecido para la finalización del Delphi ha sido el número de rondas, tal y como ya hemos apuntado, se ha calculado el análisis de estabilidad y del grado de consenso a nivel grupal, no individual para estimar la calidad de la ejecución (Landeta, 1999; Landeta, 2006; Von der Gracht, 2012).

El análisis de estabilidad se ha calculado mediante el coeficiente de variación y el rango intercuartil. Para el grado de consenso, dentro de las distintas posibilidades, se han calculado el coeficiente W de Kendall, el rango intercuartil relativo en la ronda 3 y la distribución de frecuencias. La conclusión final es que el nivel de estabilidad ha sido adecuado mientras que el grado de consenso del panel ha sido bajo aunque estadísticamente significativo.

Por otra parte, además de estudiar los resultados obtenidos en el método Delphi y el cuestionario posterior a responsables de cibercomunidades, se ha realizado un análisis comparativo de los resultados obtenidos por ambas vías en tres de las dimensiones de la investigación.

En la dimensión de las condiciones para creación y desarrollo, los resultados indican que lo obtenido por ambos procedimientos va en una dirección similar. En la Tabla 1, recogemos la distribución de las respuestas en la ronda 3 del Delphi-cCA, agrupadas por intervalos; en la tabla 2 , por su parte, se han recogido los resultados en el cuestionario-cCA.

Los ítems que parecen rechazables son los dos últimos; de hecho, no existe acuerdo en cuanto a la sincronía, e incluso se dan encuentros presenciales aunque no se organicen de modo expreso, tal y como se desprende de respuestas obtenidas a lo largo del proceso ${ }^{1}$. Por ejemplo, en el cuestionario del Delphi-cCA, indica [E24] que "me parece algo ventajoso un encuentro presencial" mientras que a juicio de [E11] "todo lo que afecta a la comunicación síncrona provoca rompimientos en la cohesión de la comunidad" y se puede constatar que se da aunque no se organice; apunta [C15] en el cuestionario-cCA que "se dan encuentros presenciales aprovechando encuentros de formación de profesores, pero no son organizados específicamente para la comunidad".

Entre los aspectos discutidos en las respuestas abiertas, además de la sincronía, tenemos el liderazgo y la moderación, el grado de apertura, si el apoyo técnico debe ser

\footnotetext{
${ }^{1}$ Entre corchetes se indica el número de la persona experta componente del panel (cuestionario DelphicCA) o el responsable de la comunidad que ha dado la respuesta (cuestionario-cCA), mediante la letra E o $\mathrm{C}$, respectivamente, seguida del número que se le ha adjudicado. Recordemos que los cuestionarios tenían preguntas cerradas y abiertas.
}

Caracterización de las cibercomunidades de aprendizaje. Iñaki Murua Anzola, Domingo J. Gallego Gil y María Luz Cacheiro González. 
interno y consciente, así como el número de miembros (Murua, 2015).

Tabla 1. Distribución de las respuestas, agrupadas por intervalos, a los ítems de la dimensión de condiciones para creación y desarrollo de cCA. Ronda 3 del Delphi-cCA.

\begin{tabular}{|c|c|c|c|}
\hline \multirow[b]{2}{*}{ ítem } & \multicolumn{3}{|c|}{ Intervalo de respuesta } \\
\hline & $8-10$ & $5-7$ & $0-4$ \\
\hline 1. Objetivos, intereses, expectativas comunes & 93,3 & 3,6 & 0 \\
\hline 2. Sentimiento de pertenencia a la comunidad & 85,7 & 14,3 & 0 \\
\hline $\begin{array}{l}\text { 3. Práctica conjunta, colaboración. participación (en interacciones conjuntas. } \\
\text { en espacios comunes...) }\end{array}$ & 78,6 & 21,4 & 0 \\
\hline $\begin{array}{l}\text { 4. Acceso a recursos compartidos (archivos de todo tipo, distintos modos de } \\
\text { comunicación síncrona y asíncrona...) }\end{array}$ & 78,6 & 21,4 & 0 \\
\hline 5. Generación colaborativa de conocimientos & $\mathbf{7 5 , 0}$ & 25,0 & 0 \\
\hline $\begin{array}{l}\text { 6. Identidad de cada miembro reconocible y estable dentro del grupo (no } \\
\text { anonimato, aunque pueda ser un nick que cambie) }\end{array}$ & 71,4 & 28,6 & 0 \\
\hline $\begin{array}{l}\text { 7. Establecimiento de compromiso (del individuo respecto al grupo y a la } \\
\text { existencia del mismo) }\end{array}$ & 69,8 & 32,1 & 0 \\
\hline $\begin{array}{l}\text { 8. Número de miembros (suficientes para que exista interacción, no } \\
\text { excesivos que la impidan) }\end{array}$ & $\mathbf{7 5 , 0}$ & 25,0 & 0 \\
\hline $\begin{array}{l}\text { 9. Existencia de distintos tipos de relaciones (de muchos a muchos, de uno a } \\
\text { muchos y de uno a uno) }\end{array}$ & 60,7 & 39,3 & 0 \\
\hline 10. Existencia de moderación / coordinación / animación & 60,7 & 39,3 & 0 \\
\hline 11. Existencia de liderazgo & 60,7 & 35,7 & 3,6 \\
\hline 12. Explicitación de normas / protocolo de comportamiento y comunicación & 57,2 & 32,1 & 0 \\
\hline 13. Capacidad del grupo para influir en las herramientas a utilizar & 49,9 & 39,2 & 7,4 \\
\hline $\begin{array}{l}\text { 14. Formación casual a partir de la experiencia y de las conexiones a Internet, } \\
\text { no dirigida por jerarquías o agentes externos interesados }\end{array}$ & 49,9 & 46,4 & 3,6 \\
\hline 15. Existencia de apoyo técnico & 50 & 32,2 & 17,9 \\
\hline 16. Estar circunscrito a un entorno / site (tipo Moodle. Ning...) & 35,3 & 39,3 & 22,2 \\
\hline $\begin{array}{l}\text { 17. Posibilidad de encuentros presenciales que complementen las relaciones } \\
\text { online }\end{array}$ & 35,7 & 35,7 & 28,6 \\
\hline $\begin{array}{l}\text { 18. Estar circunscrita a un entorno cerrado, que exija inscripción y aceptación } \\
\text { por parte de las personas responsables }\end{array}$ & 14,2 & 39,3 & 46,4 \\
\hline
\end{tabular}

//Nota. Expertos que contestan a la ronda 3, 28. Importancia concedida: 0, mínima, a 10, máxima. Destacados en negrita los valores superiores a $70 \% / /$ 
Tabla 2. Resultados sobre las condiciones que deben cumplir las cCA para su creación y desarrollo. Cuestionario-cCA.

\begin{tabular}{|c|c|c|c|c|c|}
\hline \multirow{2}{*}{ item } & & \multicolumn{4}{|c|}{$\%$} \\
\hline & & 0 & 1 & 2 & $\sqrt{3}$ \\
\hline 1. & Objetiv os, intereses y expectativas comunes & 0 & 0 & 5,6 & $\overline{94,4}$ \\
\hline 2. & Sentimiento de pertenencia a la comunidad & 0 & 0 & 38,9 & 61,1 \\
\hline $\begin{array}{l}3 . \\
\text { conjun }\end{array}$ & $\begin{array}{l}\text { Práctica conjunta, colaboración, participación (en interacciones } \\
\text { as, en espacios comunes...) }\end{array}$ & 0 & 0 & 22,2 & 77,8 \\
\hline $\begin{array}{l}4 . \\
\text { modos }\end{array}$ & $\begin{array}{l}\text { Acceso a recursos compartidos (archivos de todo tipo, distintos } \\
\text { de comunicación síncrona y asíncrona...) }\end{array}$ & 0 & 0 & 16,7 & 83,3 \\
\hline 5. & Generación colaborativa de conocimientos & 0 & 0 & 22,2 & 77,8 \\
\hline $\begin{array}{l}6 . \\
\text { (no an }\end{array}$ & $\begin{array}{l}\text { Identidad de cada miembro reconocible y estable dentro del grupo } \\
\text { nimato, aunque pueda ser un nick que no cambie) }\end{array}$ & 0 & 0 & 16,7 & 83,3 \\
\hline $\begin{array}{c}7 . \\
\text { la exis }\end{array}$ & $\begin{array}{l}\text { Establecimiento de compromiso (del individuo respecto al grupo y a } \\
\text { encia del mismo) }\end{array}$ & 0 & 22,2 & 38,9 & 38,9 \\
\hline $\begin{array}{l}8 . \\
\text { excesin }\end{array}$ & $\begin{array}{l}\text { Número de miembros (suficientes para que exista interacción, no } \\
\text { os que la impidan) }\end{array}$ & 0 & 0 & 16,7 & 83,3 \\
\hline $\begin{array}{l}9 . \\
\text { uno a } \mathrm{r}\end{array}$ & $\begin{array}{l}\text { Existencia de distintos tipos de relaciones (de muchos a muchos. de } \\
\text { uchos y de uno a uno) }\end{array}$ & 0 & 5,6 & 16,7 & 77,8 \\
\hline 10. & Existencia de moderación / coordinación / animación & 0 & 11,1 & 16,7 & 72,2 \\
\hline 11. & Existencia de liderazgo & 0 & 11,1 & 22,2 & 66,7 \\
\hline $\begin{array}{c}12 . \\
\text { comun }\end{array}$ & $\begin{array}{l}\text { Explicitación de normas / protocolo de comportamiento y } \\
\text { cación }\end{array}$ & 0 & 16,7 & 33,3 & 50,0 \\
\hline 13. & Capacidad del grupo para influir en las herramientas a utilizar & 0 & 11,1 & 44,4 & 44,4 \\
\hline $\begin{array}{l}14 . \\
\text { Inteme }\end{array}$ & $\begin{array}{l}\text { Formación casual a partir de la experiencia y de las conexiones en } \\
\text { no dirigida por jerarquías o agentes externos interesados }\end{array}$ & 5,6 & 16,7 & 27,8 & 50,0 \\
\hline & Existencia de apoyo técnico & 5,6 & 11,1 & 22,2 & 61,1 \\
\hline & Estar circunscrita a un entorno / site (tipo Moodle, Ning...) & 5,6 & 11,1 & 16,7 & 66,7 \\
\hline $\begin{array}{l}17 . \\
\text { relacior }\end{array}$ & $\begin{array}{l}\text { Posibilidad de encuentros presenciales que complementen las } \\
\text { es online }\end{array}$ & 5,6 & 27,8 & 27,8 & 38,9 \\
\hline $\begin{array}{l}18 . \\
\text { aceptac }\end{array}$ & $\begin{array}{l}\text { Estar circunscrita a un entorno cerrado, que exija inscripción y } \\
\text { ión por parte de las personas responsables }\end{array}$ & 5,6 & 38,9 & 11,1 & 44,4 \\
\hline
\end{tabular}

// Nota.- 0: no sabe - no contesta / 1: no hay constancia de su existencia / 2: hay indicios de su existencia / 3: hay evidencias de su existencia / Destacados en negrita los valores iguales o superiores a 50 //

Por el contrario, se aprecian ciertas diferencias en la dimensión de herramientas y funcionalidades. En este caso, se han recogido en la misma tabla (Tabla 3) los resultados del Delphi-cCA y del cuestionario-cCA, teniendo en cuenta que en el DelphicCA se pidió a las personas expertas que valoraran la importancia de 0 a 10 y en el cuestionario-cCA se preguntó por la existencia y uso de la herramienta en la comunidad mediante una escala tipo Likert (dando cinco opciones de respuesta: no dispone, dispone pero no se utiliza, dispone pero apenas se utiliza, dispone y se utiliza con relativa frecuencia, dispone y se utiliza de manera habitual). Asimismo, el ítem 18 con la opción de "otras herramientas" solo aparecía en el cuestionario-cCA, no en el Delphi- 
cCA, si bien como aportación de las personas expertas se introdujo desde la ronda 2 el ítem "canal de vídeo".

Tabla 3. Comparación entre resultados del Delphi-cCA y el cuestionario-cCA: herramientas y funcionalidades.

\begin{tabular}{|c|c|c|c|c|c|c|c|c|}
\hline \multicolumn{5}{|c|}{ Delphi } & \multirow[b]{2}{*}{ ÍTEM } & \multicolumn{3}{|c|}{ Cuestionario } \\
\hline $\mathrm{Q}_{1}$ & $\mathrm{Q}_{2}$ & $Q_{3}$ & Med & D.T. & & $\begin{array}{c}\% \text { Uso } \\
\text { hab. }\end{array}$ & $\begin{array}{c}\% \text { Uso } \\
\text { hab + } \\
\text { frec }\end{array}$ & $\begin{array}{l}\% \text { No } \\
\text { dispone }\end{array}$ \\
\hline 9,0 & 10 & 10 & 9,43 & 0,742 & 1. Foros & $\mathbf{5 0 , 0}$ & 66,7 & 16,7 \\
\hline 8,0 & 9,0 & 10 & 8,68 & 1,335 & 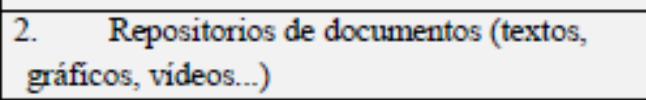 & 66,7 & 83,4 & 11,1 \\
\hline 7,25 & 8,5 & 9,0 & 8,18 & 1,565 & $\begin{array}{l}\text { 3. Espacios para colaborar, documentos } \\
\text { compartidos (tipo wiki; se entiende en sentido } \\
\text { amplio, no solo textos) }\end{array}$ & 27,8 & 38,9 & 27,8 \\
\hline 7,0 & 8,0 & 9,0 & 7,89 & 1,449 & $\begin{array}{l}\text { 4. Sistemas para guardar y compartir } \\
\text { información / curación de contenidos } \\
\text { (Delicious, Diigo, Evernote, Scoop.it ...) }\end{array}$ & 38,9 & 66,7 & 33,3 \\
\hline 7,0 & 8,0 & 9,0 & 7,79 & 2,061 & 5. $\quad$ Sistemas de microblogging (Twitter...) & $\mathbf{5 0 , 0}$ & 66,7 & 16,7 \\
\hline 6,0 & 8,0 & 9,0 & 7,75 & 1,735 & $\begin{array}{l}6 . \quad \text { Calendario / agenda / sistema para } \\
\text { concertar citas (Doodle...) }\end{array}$ & 33,3 & 44,4 & 44,4 \\
\hline 6,0 & 8,0 & 9,0 & 7,68 & 2,212 & 7. Mensajeria interna de un entorno virtual & 44,4 & 61,1 & 16,7 \\
\hline 6,0 & 8,0 & 9,0 & 7,57 & 1,834 & 8. Canal de vídeo (YouTube. Vimeo...) & 27,8 & 44,5 & 44,4 \\
\hline 7,0 & 8,0 & 9,0 & 7,50 & 2,203 & $\begin{array}{l}\text { 9. Commnicación sincrona audiovisual } \\
\text { (tipo Skype, hangouts...) }\end{array}$ & 22,2 & 44,4 & 38,9 \\
\hline 6,25 & 8,50 & 10 & 7,46 & 3,073 & 10. Correo electrónico & 44,4 & 55,5 & 33,3 \\
\hline 6,0 & 7,0 & 9,0 & 7,25 & 1,818 & 11. Sistemas para realizar encuestas online & 38,9 & $\mathbf{5 0 , 0}$ & 16,7 \\
\hline 6,0 & 7,0 & 9,0 & 7,11 & 2,439 & 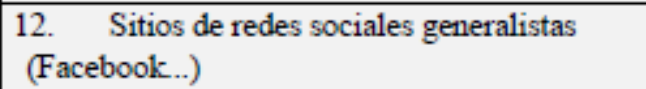 & 44,4 & 55,5 & 27,8 \\
\hline 5,0 & 7,0 & 9,0 & 6,93 & 2,124 & 13. Álbumes de fotografias / imágenes & 38,9 & 66,7 & 22,2 \\
\hline 6,0 & 7,0 & 9,0 & 6,89 & 2,470 & $\begin{array}{l}\text { 14. Sitios de redes sociales profesionales } \\
\text { (LinkedIn...) }\end{array}$ & 22,2 & 27,8 & 61,1 \\
\hline 5,25 & 8,0 & 9,0 & 6,86 & 2,889 & 15. Comunicación síncrona escrita (chat...) & 22,2 & 27,8 & 38,9 \\
\hline 5,0 & 6,50 & 8,75 & 6,64 & 2,329 & Sistemas para sindicación. RSS & 16,7 & 33,4 & 33,3 \\
\hline 5,0 & 7,50 & 9,0 & 6,64 & 3,153 & Listas de correo & 22,2 & 33,3 & $\mathbf{5 0 , 0}$ \\
\hline & & $>$ & $<$ & $>$ & Otros & & & 66,7 \\
\hline
\end{tabular}

// Nota.- Datos de la tercera ronda del Delphi-cCA / $\mathrm{Q}_{1}$ : cuartil $1 / \mathrm{Q}_{2}$ : cuartil 2 / $\mathrm{Q}_{3}$ : cuartil 3 / Med: media / D.T.: desviación típica. / \% Uso hab.: \% responsables que han respondido que la herramienta en cuestión se utiliza de manera habitual / \% Uso hab. + frec.: suma de $\%$ de responsables que han respondido que la herramienta se utiliza de forma habitual o de manera frecuente / \% No dispone: \% responsables que han respondido que la comunidad no dispone de la herramienta //

De las opiniones recogidas en las preguntas abiertas en el cuestionario Delphi-cCA, coinciden las opiniones sobre la importancia relativa de las herramientas, su número y su relación con los objetivos. A juicio de [E27], "en función del sustrato ideológico y cultural de la comunidad las herramientas pueden ser muy distintas". Indica [E7] que 
"todas las herramientas son o pueden ser necesarias, aunque ninguna imprescindible en sí misma", en lo que coincide [E25]: "todas las herramientas son importantes, pero ninguna imprescindible". En esa línea sobre herramientas, [E13] apunta que "mi pregunta es para hacer qué" y que "un número excesivo de herramientas y opciones complica más que simplifica la vida de la cibercomunidad [...] a veces, más es menos". Para [E28], "las herramientas imprescindibles son las de comunicación; para el resto, quizá sea la propia comunidad según sus objetivos y dinámica quien deba configurar su caja de herramientas (y que no sea por exceso)".

Partiendo de la tipología de participación (Murua, 2015), viene a darse una estimación similar en cuanto a porcentaje de participantes pasivos con los dos instrumentos de análisis aplicados, en torno al $40 \%$-se trata de comunidades que no están ligadas a procesos formativos en marcha, por lo que la participación es totalmente libre-. Aun así, tal y como se recoge en el Gráfico 1, en los resultados del Delphi-cCA son mayores los porcentajes estimados de personas implicadas y activas, pero menor el de ausentes.

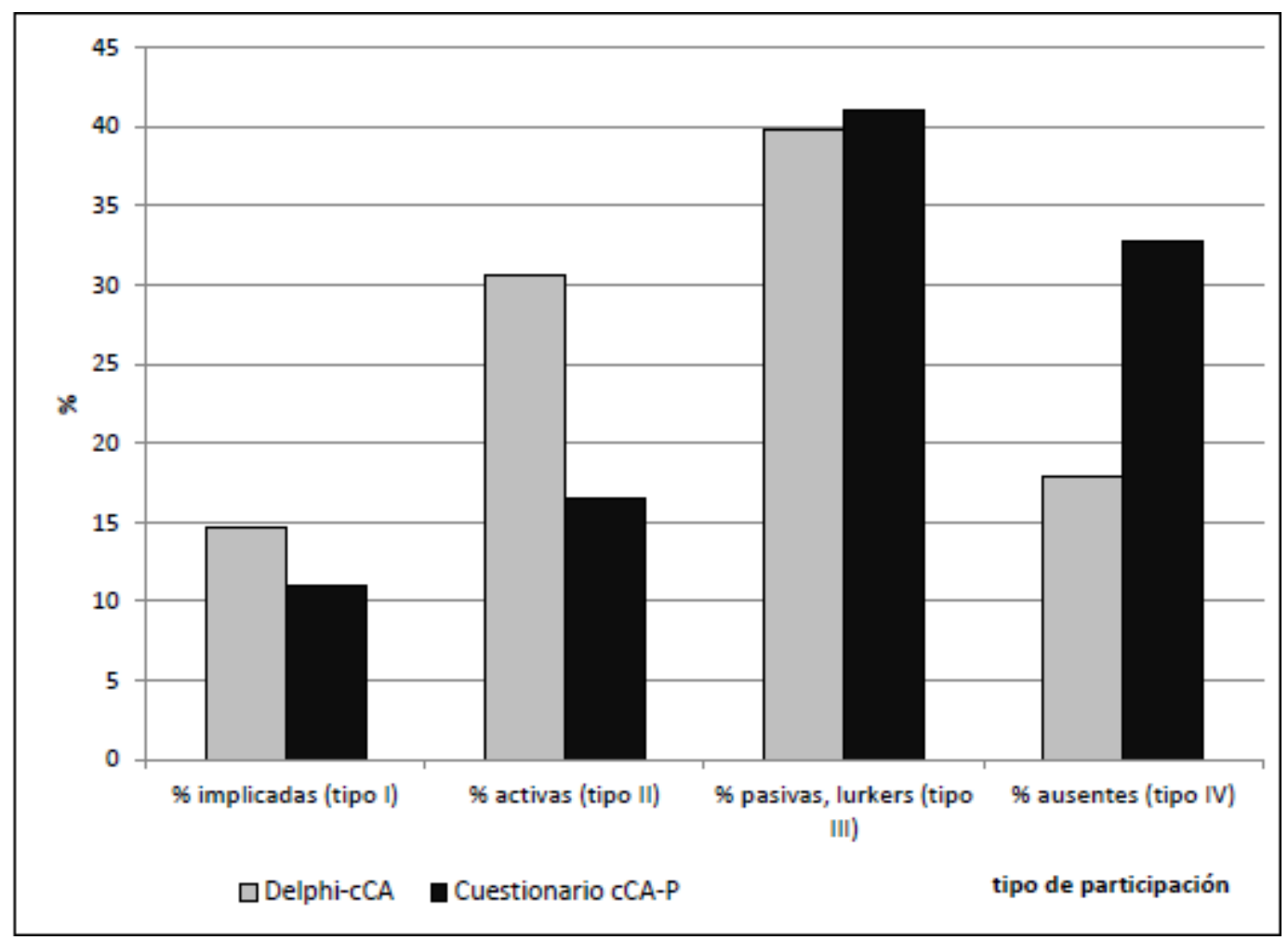

Gráfico 1. Comparación entre resultados del Delphi-cCA y el cuestionario-cCA: porcentajes de participación.

Los distintos participantes alertan, a tenor de las respuestas recogidas en los ítems de respuesta abierta de la difícil cuantificación y la variabilidad de la misma; lo explica así [E1] en el cuestionario del Delphi-cCA: "Los porcentajes pueden verse alterados por la temática tratada en un determinado momento en la comunidad. También las circunstancias personales y profesionales pueden modificar la participación de los integrantes".

A tenor de las respuestas recogidas en los ítems de respuesta abierta, varían tanto la 
consideración de quienes no participan para quienes sí lo hacen o son responsables, como las razones para participar o para no hacerlo; entre estas se menciona con cierta frecuencia la influencia del tipo de comunidad.

Por ejemplo, en el cuestionario del Delphi-cCA, a juicio de [E1], "una misma persona puede adoptar distintos roles dependiendo de la comunidad en la que participe en cada momento". Indica [E23] que "el nivel de participación depende de la actividad y del momento", y, como explica [E27], "la capacidad de atención de las personas es limitada, normalmente menor que la que ha planificado previamente". [E9] matiza que "en las primeras semanas hay muchos lurkers que pasan a ser más activos y entran de lleno en el corazón de la comunidad, otros no", indica [E13] que "los miembros nuevos llegan, participan y se comprometen o desaparecen; algunos miembros activos pasan períodos más o menos activos o lurkeando por motivos profesionales o personales, $\mathrm{y}$ luego vuelven a ser activos o desaparecen definitivamente. [E11] aclara que "[en su experiencia] las personas que no se conectan son contactadas y preguntadas por los motivos, se les ofrece ayuda si tienen problemas de conocimientos técnicos [...] Si la respuesta es que no les interesa [...] se les avisa que se les dará de baja y se les invita a volver a solicitar su ingreso si en algún momento entienden que les puede ser útil la pertenencia". Si bien también explica que "no hay que penalizar a los miembros que no son visiblemente activos durante un tiempo; a la comunidad también se acude a formarse, y hay que dar tiempo de aprender y adaptarse". Sin embargo, [E31] considera que "las personas ausentes no forman parte de la comunidad".

\section{Conclusiones}

\section{En torno a la definición de cCA}

Tras las revisiones de distintas fuentes documentales y las diversas aportaciones de los expertos y responsables de comunidades que participaron en las distintas etapas de la investigación, se propone la siguiente definición de cibercomunidad de aprendizaje (cCA): es un grupo de profesionales, estudiantes o personas con intereses comunes, vinculadas a través de Internet de forma continuada en el tiempo, para compartir información, ideas, reflexiones, experiencias, etc., con el propósito de velar por su desarrollo personal, académico y/o profesional así como el de los demás miembros.

Tal y como puede observarse, se ha matizado la definición dada al principio de la investigación. Asimismo, se ha dado un alto grado de acuerdo con esta definición, atendiendo a las respuestas del cuestionario respondido por los responsables de comunidades, independientemente del tamaño y de las características de aquellas.

En cuanto a otros aspectos, que pueden enriquecer la definición de cCA, podemos resaltar los siguientes:

- $\quad$ Las comunidades se mueven por intereses y necesidades, no por objetivos.

- No deberían desligarse de las comunidades de formación de profesionales y estar integradas en el trabajo, aunque se trate de cibercomunidades.

- $\quad$ El compromiso mutuo es imprescindible para la existencia de comunidad y debe considerarse el tipo de interacción entre los miembros. 
En definitiva, se trata de una definición que está más cercana a las comunidades de práctica, pese a que algunos participantes en el proceso investigador han insistido en la diferencia entre unas y otras. Consideramos, incluso, que con el propio uso de cibercomunidad en lugar de comunidad virtual se remarca la diferencia con el movimiento de las comunidades de práctica. Más aún; la distinción que supone utilizar cibercomunidad de aprendizaje o cCA, permite incidir más en la idea del aprendizaje no formal o informal y el aprendizaje a lo largo de toda la vida.

Asimismo, encontramos en la definición propuesta una conexión con el constructo sinergia macramental (Donaire, 2007; Fernández, 2007) en el que se juega con el poder de la representación visual del macramé -arte de hacer nudos decorativos-, y con el concepto de sinergia -la integración de elementos es más que la suma de los mismos-. En cuanto a la relación con otros conceptos en boga hoy día, como PLEs o entornos personales de aprendizaje (Castañeda y Adell, 2013) o entornos híbridos de aprendizaje personal-institucional (Álvarez, 2015), podríamos entender una cCA como el escenario de interacción que se da en la intersección de los PLEs de las personas que la componen.

Sobre las condiciones para la creación y desarrollo de una cCA en procesos formativos no formales e informales

Tras la investigación realizada, se ha reducido la lista de características y condiciones para la creación y desarrollo de cCA (Murua, Cacheiro y Gallego, 2014), quedando en dieciséis. Son las siguientes, presentadas en orden decreciente de importancia.

1. Objetivos, intereses, expectativas comunes.

2. Sentimiento de pertenencia a la comunidad.

3. Práctica conjunta, colaboración, participación (en interacciones conjuntas, en espacios comunes...).

4. Acceso a recursos compartidos (archivos de todo tipo, distintos modos de comunicación síncrona y asíncrona...).

5. Generación colaborativa de conocimientos.

6. Identidad de cada miembro reconocible y estable dentro del grupo (no anonimato, aunque pueda ser un nick que no cambie)

7. Número de miembros (suficiente para que exista interacción, no excesivo que la impida)

8. Existencia de distintos tipos de relaciones (de muchos a muchos, de uno a muchos, de uno a uno)

9. Establecimiento de compromiso (del individuo respecto al grupo y a la existencia del mismo)

10. Existencia de moderación / coordinación / animación.

11. Existencia de liderazgo.

12. Explicitación de normas / protocolo de comportamiento y comunicación.

13. Capacidad del grupo para influir en las herramientas a utilizar.

14. Formación casual a partir de la experiencia y de las conexiones a Internet, no dirigida por jerarquías o agentes externos interesados.

15. Existencia de apoyo técnico.

16. Estar basada en un entorno o site (tipo Moodle, Ning...). También puede ser considerado como tal un servicio o plataforma como Twitter. 


\section{Sobre herramientas, funcionalidades de las cCAy nivel de uso}

Como conclusión general, cabe destacar que es suficiente para la vida de una cCA la existencia de un número mínimo de herramientas que permitan la comunicación, la participación y el archivo de documentos, estén o no en un mismo entorno. Asimismo, la concreción de herramientas y funcionalidades viene determinada por los hábitos y cultura de las personas participantes. De hecho, solo tres herramientas entrarían en la categorización de importantes o muy importantes según el Delphi-cCA (foros, repositorios de documentos y espacios para colaborar tipo wiki), y esta importancia queda matizada por las respuestas obtenidas en el cuestionario-cCA, tal y como sucede con los foros, por ejemplo, cuya dificultad para construir discursos compartidos ya hemos indicado con anterioridad (Murua, 2007) o incluso su papel relevante ante otras posibilidades más audiovisuales y menos basadas en texto. Indicar, asimismo, que no se apuntan distintas herramientas respecto a la lista inicial, salvo en el caso del canal de vídeo.

Se percibe cierta problemática con los entornos cerrados frente a los abiertos, y lo que puede suponer un cambio de plataforma o las condiciones de uso, coincidente con Marín (2014). Otro fenómeno que aparece es el uso de recursos externos a los previstos en un entorno cerrado determinado, tal y como señalan de su experiencia BarreraCorominas, Fernández de Álava y Gairín (2014), que pueden ampliar los modos de comunicación, por un lado, y dispersar la conversación por otro. Otro aspecto que resulta fuente de discusión es el uso de herramientas para la comunicación síncrona, y no queda claro si puede favorecer o perjudicar a la vida de la comunidad, dependiendo, una vez más, de las características de la misma.

\section{En torno a la participación en las cCA}

La investigación realizada corrobora la existencia de una participación asimétrica, tanto con una perspectiva sincrónica como diacrónica. En todo caso, cuando no existe obligatoriedad de participar es importante el porcentaje de participación pasiva, o incluso ausente, en los casos en los que la normas -tácitas o expresas de la cCA-, así lo admiten.

Entre las causas que se apuntan para la participación pasiva están (Murua, 2015):

- Los niveles de aprendizaje distintos de los miembros.

- $\quad$ La disponibilidad de tiempo y la situación personal.

- $\quad$ La actitud de los miembros nuevos.

- $\quad$ El momento en que se encuentre la comunidad.

- La tipología de la comunidad.

Un aspecto de interés, que ha sido apuntado en el proceso investigador, se refiere a la calidad de la participación, no solo a la cantidad. La participación, en este sentido, puede ser también de tipo social, con mensajes de poco peso comunicativo y que no contribuya al objetivo de aprendizaje; en otras palabras, puede tratarse de ruido. En comunidades basadas en entornos abiertos, como pueda ser Twitter, se ha referido la aparición de mensajes que podrían calificarse de propaganda o spam.

Caracterización de las cibercomunidades de aprendizaje. Iñaki Murua Anzola, Domingo J. Gallego Gil y María Luz Cacheiro González. 


\section{Claves para una propuesta de cCA para la formación del profesorado en contextos no formales e informales}

Tras el análisis de los resultados de la investigación desarrollada se presenta una propuesta para una cCA en el ámbito de la formación del profesorado en contexto no formal / informal. Se trata de una cCA abierta cuyos potenciales destinatarios son profesores de enseñanza de euskera a personas adultas, con especial incidencia en el profesorado de los centros homologados de la Comunidad Autónoma del País Vasco.

El entorno básico, siguiendo el criterio apuntado en el párrafo anterior en cuanto a apertura, sería Twitter, con una propuesta concreta y consensuada de hashtag, sin descartar otras herramientas, como pudiera ser un espacio que sirviera de repositorio. Se trataría de buscar o detectar en ese entorno tuitero potenciales miembros que puedan formar parte como miembros activos o incluso como parte del núcleo de la cCA -personas implicadas, según la clasificación sobre participación-, si bien se trataría de atender también a las características y competencia digital de los posibles integrantes. Salvando las distancias, dos posibles ejemplos a seguir serían las comunidades establecidas en torno a los hahstags \#TwitterELE (Rastrero y Varo, 2014) e \#InstagramELE (Martín Bosque y Munday, 2014).

\section{Mirando al futuro}

Entre las líneas que nos abre la presente investigación, podemos destacar posibilidades de futuro tales como:

- Elaborar una rúbrica para evaluar cibercomunidades, basada en el cuestionariocCA utilizado, de modo similar a lo que plantea Morrison (2015).

- Identificar indicadores de éxito, cuantitativos y cualitativos, para una

- Analizar la aplicabilidad del modelo propuesto por Martínez Marín (2014) para detectar posibles líderes y/o moderadores de cara a lanzar una cCA, así como de la propuesta de Llambí (2015) para analizar personas en Twitter.

- Realizar un estudio de caso con una comunidad -o microcomunidad de entornos más grandes- del ámbito de la formación del profesorado.

- Analizar la aplicabilidad de la propuesta de Esteve (2015) sobre principios para el diseño de entornos 3D para el desarrollo y evaluación de competencias, entre ellas la digital, en el contexto del profesorado de lenguas.

- Materializar la propuesta de cCA en el entorno del profesorado de euskera a personas adultas.

Presentación del artículo: 20 de septiembre de 2015

Fecha de aprobación: 27 de septiembre de 2015

Fecha de publicación: 30 de octubre de 2015

Murua, I., Gallego, D. J. y Cacheiro, M. L. (2015). Caracterización de las cibercomunidades de aprendizaje (cCA). RED, Revista de Educación a Distancia, 47(4). 30 de octubre de 2015. Consultado el [dd/mm/aaaa] en http://www.um.es/ead/red/47/ 


\section{Referencias}

Álvarez, D. (2015, 6 de abril). Una mirada a los PLE desde las organizaciones. [Entrada de blog]. Recuperado de http://e-aprendizaje.es/2015/04/06/una-mirada-a-los-pledesde-las-organizaciones/

Barrera-Corominas, A., Fernández de Álava, M. \& Gairín, J. (2014). Aprendizaje colaborativo en comunidades de práctica online: La Plataforma e-Catalunya. EDUTEC, Revista Electrónica de Tecnología Educativa, 47, 1-13. Recuperado de http://edutec.rediris.es/Revelec2/Revelec47/n47_Barrera-Fernandez-Garin.html

Blasco, J. E., López Padrón, A. \& Mengual, S. (2010). Validación mediante método Delphi de un cuestionario para conocer las experiencias e interés hacia las actividades acuáticas con especial atención al windsurf. Ágora para la educación física y el deporte, 12 (1), 75-96. Recuperado de http://www5.uva.es/agora/revista/12_1/agora_12_1d_blasco_et_al.pdf

Cabero, J. (2014). Formación del profesorado universitario en TIC. Aplicación del método Delphi para la selección de los contenidos formativos. Educación XX1 17(1), 111-132. doi: 10.5944/educxx 1.17.1.107017

Cabero, J. \& Barroso, J. (2013). La utilización del juicio de experto para la evaluación de TIC: el coeficiente de competencia experta. Bordón, 65(2), 25-38.

Cabero, J. \& Llorente, M. C. (2013). La aplicación del juicio de experto como técnica de evaluación de las tecnologías de la información y comunicación (TIC). Revista de Tecnología de Información y Comunicación en Educación, 7(2), 11-22. Recuperado de http://servicio.bc.uc.edu.ve/educacion/eduweb/v7n2/art01.pdf

Castañeda, L. \& Adell, J. (2013). La anatomía de los PLEs. En L. Castañeda y J. Adell (eds.), Entornos Personales de Aprendizaje: Claves para el ecosistema educativo en red (pp. 11-27). Alcoy: Marfil.

Cohen, L. \& Manion, L. (1990). Métodos de investigación educativa. Madrid: La Muralla.

Coll, C., Bustos, A. \& Engel, A. (2008). Las comunidades virtuales de aprendizaje. En C. Coll \& C. Monereo (eds.), Psicología de la educación virtual (pp. 299-320). Madrid: Morata.

Domínguez Figaredo, D. (2003). Investigación educativa en contextos tecnológicos: apropiación metodológica de las nuevas tecnologías. En S. Castillo \& A. Medina (coords.), Metodología para la realización de proyectos de investigación y tesis doctorales (pp. 267-289). Madrid: Universitas.

Donaire, J. A. (2007, 6 de noviembre). Sinergia macramental. [Entrada de blog]. Recuperado de http://don-aire.blogspot.com.es/2007/11/sinergia-macramental.html 
Draper, D.C.(2015). Collaborative Instructional Strategies to Enhance Knowledge Convergence. The American Journal of Distance Education, 29(2), 109-125.

Escudero, J.M. (2009). Comunidades docentes de aprendizaje, formación del profesorado y mejora de la educación. Agora para la EF y el Deporte, 10, 7-31. Recuperado de http://www5.uva.es/agora/revista/10/agora10_escudero.pdf

Esteve, F. (2015). La competencia digital docente. Análisis de la autopercepción y evaluación del desempeño de los estudiantes universitarios de educación por medio de un entorno 3D. [Tesis doctoral]. Universitat Rovira y Virgili, Tarragona.

Fernández, L. (2007, 4 de noviembre). Sinergia macramental, el origen del palabro. [Entrada de blog]. Recuperado de http://blog.loretahur.net/2007/11/sinergiamacramental-el-origen-del-palabro.html

Flecha, R. \& Larena, R. (2008). Comunidades de aprendizaje. Sevilla: Fundación ECOEM.

García Aretio, L. (2003). Las comunidades de aprendizaje en entornos virtuales. La comunidad iberoamericana de la CUED. En M. Barajas (coord.), La tecnología educativa en la enseñanza superior (pp. 171-199). Madrid: McGraw-Hill.

Gros. B., García, I. \& Lara, P. (2009). El desarrollo de herramientas de apoyo para el trabajo colaborativo en entornos virtuales de aprendizaje. RIED, 2 (2), 115-138.

Hernández Sampieri, R.; Fernández Collado, C. \& Baptista, P. (2010). Metodología de la investigación. México D.F.: Mc Graw-Hill.

Landeta, J. (1999). El método delphi: una técnica de previsión para la incertidumbre. Barcelona: Ariel.

Landeta, J. (2006). Current validity of the Delphi method in social sciences. Technological Forecasting \& Social Change, 73, 467-482.

Luna, P., Infante, A., Martínez López, F. J. \& García Ordaz, M. (2004). Los Sistemas de Información y Tecnologías de la Información (SI/TIC) y la metodología Delphi. Virtual Educa Barcelona 2004. Recuperado de http://espacio.uned.es/fez/view.php?pid=bibliuned:19539

Llambí, P. (2015, 16 de febrero). Cómo hacer un estudio completo de tu competencia en Twitter. [Entrada de blog]. Recuperado de http://tiempodenegocios.com/como-hacerun-estudio-completo-de-tu-competencia-en-twitter/

Marín, V. (2014). Las redes sociales y la universidad. El caso de DIPRO 2.0. Revista Iberoamericana de Educación, 66(2), 1-11. 
Martín Bosque, A. \& Munday, P. (2014). Conexión, colaboración y aprendizaje más allá del aula: \#Instagramele. Actas del II Congreso Internacional Nebrija en Lingüística Aplicada a la Enseñanza de Lenguas (pp. 381-390). Recuperado de http://www.nebrija.com/la_universidad/servicios/pdfpublicaciones/ActasNebrija_SegundoCongreso.pdf

Martínez Marín, J. (2014, 22 de octubre). Dinamizadores de aprendizaje informal en 8 pasos. [Entrada de blog]. Recuperado de https://trabajocolaborativoenred.wordpress.com/2014/10/22/dinamizadores-deaprendizaje-informal-en-8-pasos/

Morrison, D. (2015, 21 de enero). How Interactive is Your Online Course? Self-Assess with this Rubric. [Entrada de blog] Recuperado de https://onlinelearninginsights.wordpress.com/2015/01/21/how-interactive-is-youronline-course-self-assess-with-this-rubric/

Murua, I. (2007). Comunidades virtuales de aprendizaje: entre las posibilidades y las dudas. IV Congreso EducaRed. doi: 10.13140/2.1.3873.5687

Murua, I. (2015). Las cibercomunidades de aprendizaje y la formación del profesorado. [Tesis doctoral]. Universidad Nacional de Educación a Distancia, Madrid. doi: 10.13140/RG.2.1.3542.3848

Murua, I., Cacheiro, M. L. \& Gallego, D. J. (2014). Las cibercomunidades de aprendizaje (cCA) en la formación del profesorado. RED Revista de Educación a Distancia, 43, 1-29. Recuperado de http://www.um.es/ead/red/43/gallego_et_al.pdf

Murua, I. y Gallego, D. J. (2014). La participación en las cibercomunidades de aprendizaje. En M. L. Cacheiro (coord.), Educación y Tecnología: Estrategias didácticas para la integración de las TIC (pp. 192-208). Madrid: UNED.

Park, J.Y. (2015).Student interactivity and teacher participation: an application of legitimate peripheral participation in higher education online learning environments. Technology, Pedagogy and Education, 24(3), 389-406. doi:10.1080/1475939X.2014.935743

Rastrero, M. \& Varo, D. (2014). \#Twitterele, historia de un hashtag. En R. Cuadros \& J. Villatoro (edit. y coords.). Twitter en la enseñanza y el aprendizaje del español (pp. 85-106). Málaga: Ediciones EdiEle. Recuperado de http://digitalingua.com/biblioteca/twitter/

Rheingold, H. (1996). La comunidad virtual. Una sociedad sin fronteras. Barcelona: Gedisa

Rovai, A. P. (2002). Building sense of community at a distance. The International Review of Research in Open and Distance Learning, 3(1), 1-16. Recuperado de http://www.irrodl.org/index.php/irrodl/article/view/79/152 
Tirado, R. y Martínez, J.M. (2009). Creando comunidades virtuales de aprendizaje: análisis del progreso de las interacciones. Revista de Educación, 353, 297-328. Recuperado de http://www.ince.mec.es/revistaeducacion/re353/re353_11.pdf

Von der Gracht, H. A. (2012). Consensus measurement in Delphi studies. Review and implications for future quality assurance. Technological Forecasting \& Social Change, 79(8), 1525-1636. doi: 10.1016/j.techfore.2012.04.013 